ISSN 1600-5368

\section{Crystal structure of hydrocortisone 17-butyrate}

\section{Yu Zhu, ${ }^{*}$ Wei Shen and Hai-li Wang}

College of Chemistry and Molecular Engineering, Zhengzhou University, Zhengzhou 450001, People's Republic of China. *Correspondence e-mail: zhuyu@zzu.edu.cn

Received 21 October 2014; accepted 30 October 2014

Edited by C. Rizzoli, Universita degli Studi di Parma, Italy

In the title compound, $\mathrm{C}_{25} \mathrm{H}_{36} \mathrm{O}_{6}$, the two central cyclohexane rings exhibit a chair conformation. The terminal cyclohexene and cyclopentane rings are in half-chair and envelope conformations (with the $\mathrm{C}$ atom bearing the methyl substituent as the flap), respectively. The methyl group of the butyrate chain is disordered over two orientations, with a refined occupancy ratio of 0.742 (6):0.258 (6). Intramolecular $\mathrm{O}-\mathrm{H} \cdots \mathrm{O}$ and $\mathrm{C}-\mathrm{H} \cdots \mathrm{O}$ hydrogen bonds are observed. In the crystal, molecules are linked by $\mathrm{O}-\mathrm{H} \cdots \mathrm{O}$ hydrogen bonds into chains running parallel to the $a$ axis.

Keywords: crystal structure; hydrocortisone derivative; hydrogen bonds; pharmacological activity.

CCDC reference: 1031721

\section{Related literature}

For the pharmacological activities of the title compound, see: Haapasaari et al. (1995); Lerche et al. (2010); D'Erme \& Gola (2012). For the synthesis of the title compound, see: Sun et al. (2009).

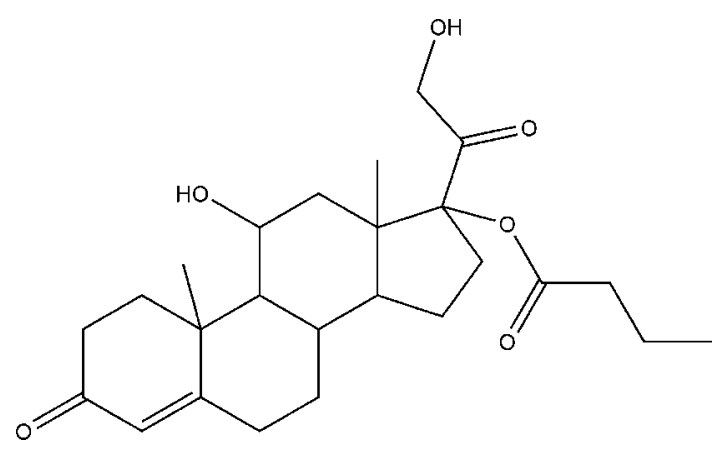

\section{Experimental}

\subsection{Crystal data}

$\mathrm{C}_{25} \mathrm{H}_{36} \mathrm{O}_{6}$

Orthorhombic, $P 2_{1} 2_{1} 2_{1}$

$a=9.05738(8) \AA$

$b=11.87633$ (9) $\AA$

$c=21.13465(15) \AA$

$$
\begin{aligned}
& V=2273.42(3) \AA^{3} \\
& Z=4 \\
& \mathrm{Cu} K \alpha \text { radiation } \\
& \mu=0.72 \mathrm{~mm}^{-1} \\
& T=291 \mathrm{~K} \\
& 0.22 \times 0.2 \times 0.2 \mathrm{~mm}
\end{aligned}
$$

\subsection{Data collection}

Agilent Xcalibur Eos Gemini diffractometer

Absorption correction: multi-scan (CrysAlis PRO (Agilent, 2011)

$T_{\min }=0.952, T_{\max }=1.000$

23353 measured reflections 4496 independent reflections 4389 reflections with $I>2 \sigma(I)$ $R_{\text {int }}=0.021$

\subsection{Refinement}

$R\left[F^{2}>2 \sigma\left(F^{2}\right)\right]=0.040$

$w R\left(F^{2}\right)=0.111$

$S=1.06$

4496 reflections

301 parameters

4 restraints

$\mathrm{H}$ atoms treated by a mixture of independent and constrained refinement

Table 1

Hydrogen-bond geometry $\left(\AA{ }^{\circ}\right)$.

\begin{tabular}{lllll}
\hline$D-\mathrm{H} \cdots A$ & $D-\mathrm{H}$ & $\mathrm{H} \cdots A$ & $D \cdots A$ & $D-\mathrm{H} \cdots A$ \\
\hline $\mathrm{C} 19-\mathrm{H} 19 C \cdots \mathrm{O} 2$ & 0.96 & 2.39 & $3.016(2)$ & 122 \\
$\mathrm{O} 4-\mathrm{H} 4 \cdots \mathrm{O} 3$ & $0.83(3)$ & $2.06(3)$ & $2.629(3)$ & $126(2)$ \\
$\mathrm{O} 2-\mathrm{H} 2 \cdots \mathrm{O} 1^{\mathrm{i}}$ & 0.82 & 2.11 & $2.9192(18)$ & 169 \\
\hline
\end{tabular}

Symmetry code: (i) $x+1, y, z$.

Data collection: CrysAlis PRO (Agilent, 2011); cell refinement: CrysAlis PRO; data reduction: CrysAlis PRO; program(s) used to solve structure: SHELXS97 (Sheldrick, 2008); program(s) used to refine structure: SHELXL97 (Sheldrick, 2008); molecular graphics: OLEX2 (Dolomanov et al., 2009); software used to prepare material for publication: $O L E X 2$.

\title{
Acknowledgements
}

The authors thank Ms L. R. Yang for technical assistance. This research was supported by the National Natural Science Foundation of China (No. JI210060).

Supporting information for this paper is available from the IUCr electronic archives (Reference: RZ5136).

\section{References}

Agilent (2011). CrysAlis PRO. Agilent Technologies UK Ltd, Yarnton, England.

D'Erme, A. M. \& Gola, M. (2012). Dermatitis, 23, 95-96.

Dolomanov, O. V., Bourhis, L. J., Gildea, R. J., Howard, J. A. K. \& Puschmann, H. (2009). J. Appl. Cryst. 42, 339-341.

Flack, H. D. (1983). Acta Cryst. A39, 876-881. 


\section{data reports}

Haapasaari, K. M., Risteli, J., Koivukangas, V. \& Oikarinen, A. (1995). Acta Derm. Venereol. 75, 269-271.

Lerche, C. M., Philipsen, P. A., Poulsen, T. \& Wulf, H. C. (2010). Exp. Dermatol. 19, 973-979.
Sheldrick, G. M. (2008). Acta Cryst. A64, 112-122.

Sun, L., Chen, S. \& Zhao, L. (2009). China Patent CN 101812108A. 


\section{supporting information}

Acta Cryst. (2014). E70, o1239-o1240］doi:10.1107/S1600536814023903]

\section{Crystal structure of hydrocortisone 17-butyrate}

\section{Yu Zhu, Wei Shen and Hai-li Wang}

\section{S1. Comment}

Hydrocortisone 17-butyrate is an important cortical hormone drug derived from the esterification reaction of hydrocortisone at the hydroxyl group of C-17. Due to the introduction of the alkyl chain, hydrocortisone 17-butyrate showed increased lipophicity and affinity for receptors, which lead to increased pharmacological activity (Haapasaari et al., 1995; Lerche et al., 2010; D'Erme \& Gola, 2012). Compared with hydrocortisone, it showed increased anti-inflammatory activity, immunosuppressive properties, and low side effect. Due to the outstanding characteristics of hydrocortisone 17butyrate, it has drawn great attention of the experts from the fields of chemistry, pharmacy and medicine. The synthesis and properties have been investigated quite extensively, while its molecular structure has not been reported. Here we present the single-crystal X-ray diffraction study of hydrocortisone 17-butyrate.

The molecular structure of the title compound is shown in Figure 1. As expected, the chiral carbon atoms C8, C9, C11, $\mathrm{C} 13$, and $\mathrm{C} 14$ exhibit $S$ configuration, and atoms $\mathrm{C} 10$ and $\mathrm{C} 17$ exhibit $R$ configuration. The Flack parameter is 0.02 (18). Both central six membered rings ( $\mathrm{C} 5, \mathrm{C} 6, \mathrm{C} 7, \mathrm{C} 8, \mathrm{C} 9, \mathrm{C} 10$, and $\mathrm{C} 8, \mathrm{C} 9, \mathrm{C} 11, \mathrm{C} 12, \mathrm{C} 13, \mathrm{C} 14)$ exhibit chair conformation, with atoms C5 and C8 displaced by 0.6024 (16) and 0.6378 (15) $\AA$ on opposite sides from the C6, C7, C9 and C10 plane, and atoms $\mathrm{C} 9$ and $\mathrm{C} 13$ by 0.6249 (14) and 0.7014 (15) $\AA$ from the $\mathrm{C} 8, \mathrm{C} 11, \mathrm{C} 12$ and $\mathrm{C} 14$ plane. The cyclohexene ring $(\mathrm{C} 1, \mathrm{C} 2, \mathrm{C} 3, \mathrm{C} 4, \mathrm{C} 5, \mathrm{C} 10)$ assumes a half-chair conformation, atom $\mathrm{C} 2$ protruding by 0.534 (2) $\AA$ from the mean plane through the remaining five atoms. The cyclopentane ring is in an envelope conformation, with atom $\mathrm{C} 13$ displaced by 0.6761 (15) $\AA$ from the $\mathrm{C} 14, \mathrm{C} 15, \mathrm{C} 16, \mathrm{C} 17$ mean plane. Intramolecular $\mathrm{O}-\mathrm{H} \cdots \mathrm{O}$ and $\mathrm{C}-\mathrm{H} \cdots \mathrm{O}$ hydrogen bonds are observed (Table 1). In the crystal, molecules are connected by intermolecular $\mathrm{O}-\mathrm{H} \cdots \mathrm{O}$ hydrogen bonds to form chains parallel to the $a$ axis.

\section{S2. Experimental}

The title compound was obtained following a patent report (Sun et al., 2009). At 0-5 ${ }^{\circ} \mathrm{C}$, butyryl chloride (1.5 mmol, 0.16 $\mathrm{mL}$ ) was added dropwise to a $\mathrm{CH}_{2} \mathrm{Cl}_{2}(10 \mathrm{~mL})$ solution containing hydrocortisone 21 -acetate (1 mmol, $\left.0.4 \mathrm{~g}\right), \mathrm{Et}_{3} \mathrm{~N}(4$ $\mathrm{mL}$ ) and 4-dimethylaminopyridine $(0.05 \mathrm{mmol}, 6 \mathrm{mg})$. The mixture was then stirred at $0^{\circ} \mathrm{C}$ for 3 hours before being treated with $\mathrm{HCl}$ to reach a $\mathrm{pH}$ of 2 . The mixture was then washed with $\mathrm{H}_{2} \mathrm{O}$ to reach neutrality, and extracted using $\mathrm{CH}_{2} \mathrm{Cl}_{2}$. The organic phase was combined, dried, and evaporated. Crystallization of the residue in $\mathrm{MeOH}$ produced hydrocortisone 17-butyrate 21-acetate. The $\mathrm{CH}_{2} \mathrm{Cl}_{2}$ solution of hydrocortisone 17-butyrate 21-acetate was added slowly to a $\mathrm{MeOH}(10 \mathrm{~mL})$ solution of $\mathrm{K}_{2} \mathrm{CO}_{3}(0.1 \mathrm{~g})$ at $-10^{\circ} \mathrm{C}$ for selective hydrolysis. The mixture was then neutralized by $\mathrm{CH}_{3} \mathrm{COOH}$, washed with $\mathrm{H}_{2} \mathrm{O}$, and extracted with $\mathrm{CH}_{2} \mathrm{Cl}_{2}$. The organic phase was combined, dried and evaporated. Crystallization of the residue in $\mathrm{MeOH}$ at $0^{\circ} \mathrm{C}$ produced pure hydrocortisone 17-butyrate. Crystals suitable for X-ray analysis were obtained by slow evaporation of a $\mathrm{MeOH}(20 \mathrm{~mL})$ solution of hydrocortisone 17-butyrate $(5 \mathrm{mg})$ at room temperature. 


\section{S3. Refinement}

The $\mathrm{C} 25$ methyl carbon atom is disordered over two orientations with refined occupancy ratio 0.742 (6):0.258 (6). The disordered atoms were refined by constraining the C24-C25 and C24-C25A bond lengths to be 1.52 (1) $\AA$ and by restraining the anisotropic displacement ellipsoids to be equal. The hydroxyl $\mathrm{H}$ atom bound to $\mathrm{O} 4$ was located in a difference Fourier map and refined freely. All other $\mathrm{H}$ atoms were placed in calculated positions and refined as riding, with $\mathrm{C}-\mathrm{H}=0.93-0.97 \AA, \mathrm{O}-\mathrm{H}=0.82 \AA$, and with $U_{\mathrm{iso}}(\mathrm{H})=1.2 U_{\mathrm{eq}}(\mathrm{C})$ or $1.5 U_{\mathrm{eq}}(\mathrm{C})$ for methyl and hydroxyl $\mathrm{H}$ atoms. A rotating model was applied to the methyl and hydroxyl groups. One outlier $\left(\begin{array}{lll}0 & 1 & 1\end{array}\right)$ was omitted in the last cycles of refinement.

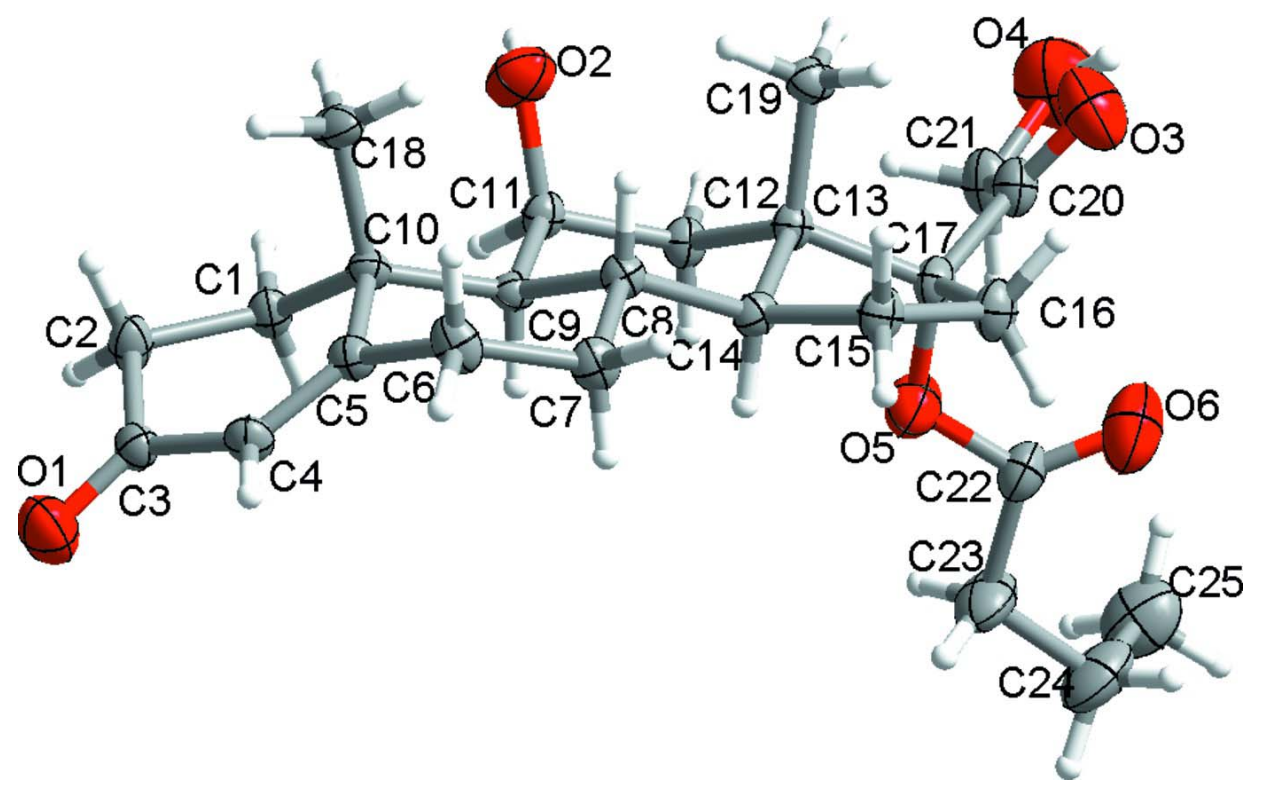

\section{Figure 1}

The molecular structure of the title compound showing 30\% probability displacement ellipsoids. Only the major component of the disordered C25 methyl group is shown

(8S,9S, 10R, 11S, 13S, 14S,17R)-11-Hydroxy-17-(2-hydroxyacetyl)-10,13-dimethyl-3-

oxo-2,6,7,8,9,11,12,14,15,16-decahydro-1H-cyclopenta[a]phenanthren-17-yl butanoate

Crystal data

$\mathrm{C}_{25} \mathrm{H}_{36} \mathrm{O}_{6}$

$M_{r}=432.54$

Orthorhombic, $P 2{ }_{1} 2{ }_{1} 2_{1}$

$a=9.05738(8) \AA$

$b=11.87633(9) \AA$

$c=21.13465(15) \AA$

$V=2273.42(3) \AA^{3}$

$Z=4$

$F(000)=936$

\section{Data collection}

Agilent Xcalibur Eos Gemini diffractometer

Radiation source: Enhance (Cu) X-ray Source Graphite monochromator
$D_{\mathrm{x}}=1.264 \mathrm{Mg} \mathrm{m}^{-3}$

$\mathrm{Cu} K \alpha$ radiation, $\lambda=1.54184 \AA$

Cell parameters from 14007 reflections

$\theta=4.2-72.4^{\circ}$

$\mu=0.72 \mathrm{~mm}^{-1}$

$T=291 \mathrm{~K}$

Block, colourless

$0.22 \times 0.2 \times 0.2 \mathrm{~mm}$

Detector resolution: 16.2312 pixels $\mathrm{mm}^{-1}$ $\omega$ scans

Absorption correction: multi-scan

(CrysAlis PRO (Agilent, 2011) 
$T_{\min }=0.952, T_{\max }=1.000$

23353 measured reflections

4496 independent reflections

4389 reflections with $I>2 \sigma(I)$

$R_{\text {int }}=0.021$

\section{Refinement}

Refinement on $F^{2}$

Least-squares matrix: full

$R\left[F^{2}>2 \sigma\left(F^{2}\right)\right]=0.040$

$w R\left(F^{2}\right)=0.111$

$S=1.06$

4496 reflections

301 parameters

4 restraints

Primary atom site location: structure-invariant direct methods

Secondary atom site location: difference Fourier map

$$
\begin{aligned}
& \theta_{\text {max }}=72.3^{\circ}, \theta_{\text {min }}=4.2^{\circ} \\
& h=-9 \rightarrow 11 \\
& k=-14 \rightarrow 14 \\
& l=-26 \rightarrow 26
\end{aligned}
$$

Hydrogen site location: inferred from neighbouring sites

$\mathrm{H}$ atoms treated by a mixture of independent and constrained refinement

$w=1 /\left[\sigma^{2}\left(F_{\mathrm{o}}^{2}\right)+(0.0712 P)^{2}+0.3126 P\right]$ where $P=\left(F_{\mathrm{o}}^{2}+2 F_{\mathrm{c}}^{2}\right) / 3$

$(\Delta / \sigma)_{\max }=0.001$

$\Delta \rho_{\max }=0.31$ e $\AA^{-3}$

$\Delta \rho_{\min }=-0.28$ e $\AA^{-3}$

Absolute structure: Flack (1983), 1932 Friedel pairs

Absolute structure parameter: 0.02 (18)

\section{Special details}

Geometry. All e.s.d.'s (except the e.s.d. in the dihedral angle between two 1.s. planes) are estimated using the full covariance matrix. The cell e.s.d.'s are taken into account individually in the estimation of e.s.d.'s in distances, angles and torsion angles; correlations between e.s.d.'s in cell parameters are only used when they are defined by crystal symmetry. An approximate (isotropic) treatment of cell e.s.d.'s is used for estimating e.s.d.'s involving 1.s. planes.

Refinement. Refinement of $F^{2}$ against ALL reflections. The weighted $R$-factor $w R$ and goodness of fit $S$ are based on $F^{2}$, conventional $R$-factors $R$ are based on $F$, with $F$ set to zero for negative $F^{2}$. The threshold expression of $F^{2}>\sigma\left(F^{2}\right)$ is used

\begin{tabular}{|c|c|c|c|c|c|}
\hline & $x$ & $y$ & $z$ & $U_{\text {iso }} * / U_{\text {eq }}$ & Occ. $(<1)$ \\
\hline $\mathrm{O} 1$ & $-0.36156(14)$ & $0.73133(12)$ & $0.93301(6)$ & 0.0499 & \\
\hline $\mathrm{O} 2$ & $0.38389(14)$ & $0.61706(12)$ & $0.87774(6)$ & 0.0468 & \\
\hline $\mathrm{H} 2$ & 0.4512 & 0.6572 & 0.8906 & $0.070 *$ & \\
\hline $\mathrm{O} 3$ & $0.70484(17)$ & $0.51666(13)$ & $0.64786(8)$ & $0.0624(4)$ & \\
\hline O4 & $0.8532(2)$ & $0.7026(2)$ & $0.67034(11)$ & $0.0791(5)$ & \\
\hline $\mathrm{H} 4$ & $0.866(3)$ & $0.638(2)$ & $0.6577(13)$ & $0.059(7)^{*}$ & \\
\hline O5 & $0.39480(15)$ & $0.69247(10)$ & $0.63513(5)$ & 0.0448 & \\
\hline O6 & $0.5163(2)$ & $0.66663(14)$ & $0.54407(7)$ & $0.0670(4)$ & \\
\hline $\mathrm{C} 1$ & $0.02943(18)$ & $0.72375(15)$ & $0.90894(8)$ & $0.0409(3)$ & \\
\hline $\mathrm{H} 1 \mathrm{~A}$ & 0.1177 & 0.7472 & 0.9314 & $0.049 *$ & \\
\hline H1B & 0.0142 & 0.7759 & 0.8743 & $0.049 *$ & \\
\hline $\mathrm{C} 2$ & $-0.1022(2)$ & $0.73130(18)$ & $0.95395(9)$ & $0.0483(4)$ & \\
\hline $\mathrm{H} 2 \mathrm{~A}$ & -0.0831 & 0.6860 & 0.9912 & $0.058 *$ & \\
\hline $\mathrm{H} 2 \mathrm{~B}$ & -0.1154 & 0.8087 & 0.9674 & $0.058^{*}$ & \\
\hline $\mathrm{C} 3$ & $-0.24009(18)$ & $0.69069(14)$ & $0.92219(7)$ & $0.0391(3)$ & \\
\hline $\mathrm{C} 4$ & $-0.22232(18)$ & $0.59526(15)$ & $0.87958(8)$ & $0.0408(3)$ & \\
\hline $\mathrm{H} 4 \mathrm{~A}$ & -0.3071 & 0.5621 & 0.8632 & $0.049 *$ & \\
\hline $\mathrm{C} 5$ & $-0.09161(18)$ & $0.55240(13)$ & $0.86262(7)$ & $0.0373(3)$ & \\
\hline C6 & $-0.0827(2)$ & $0.44526(14)$ & $0.82502(9)$ & $0.0453(4)$ & \\
\hline
\end{tabular}
only for calculating $R$-factors(gt) etc. and is not relevant to the choice of reflections for refinement. $R$-factors based on $F^{2}$ are statistically about twice as large as those based on $F$, and $R$-factors based on ALL data will be even larger.

Fractional atomic coordinates and isotropic or equivalent isotropic displacement parameters $\left(\AA^{2}\right)$ 


\begin{tabular}{|c|c|c|c|c|c|}
\hline H6A & -0.1814 & 0.4225 & 0.8126 & $0.054^{*}$ & \\
\hline H6B & -0.0415 & 0.3862 & 0.8513 & $0.054 *$ & \\
\hline $\mathrm{C} 7$ & $0.0122(2)$ & $0.45906(15)$ & $0.76617(9)$ & $0.0440(4)$ & \\
\hline $\mathrm{H} 7 \mathrm{~A}$ & -0.0378 & 0.5087 & 0.7367 & $0.053^{*}$ & \\
\hline $\mathrm{H} 7 \mathrm{~B}$ & 0.0237 & 0.3864 & 0.7458 & $0.053^{*}$ & \\
\hline $\mathrm{C} 8$ & $0.16479(17)$ & $0.50722(13)$ & $0.78101(7)$ & $0.0336(3)$ & \\
\hline $\mathrm{H} 8$ & 0.2217 & 0.4516 & 0.8051 & $0.040^{*}$ & \\
\hline C9 & $0.15146(16)$ & $0.61680(12)$ & $0.82020(7)$ & $0.0311(3)$ & \\
\hline H9 & 0.0957 & 0.6684 & 0.7930 & $0.037^{*}$ & \\
\hline $\mathrm{C} 10$ & $0.05504(16)$ & $0.60493(14)$ & $0.88151(7)$ & 0.0339 & \\
\hline $\mathrm{C} 11$ & $0.30159(18)$ & $0.67602(13)$ & $0.83072(7)$ & $0.0355(3)$ & \\
\hline H11 & 0.2813 & 0.7517 & 0.8471 & $0.043^{*}$ & \\
\hline $\mathrm{C} 12$ & 0.39077 (18) & $0.68924(12)$ & $0.76887(7)$ & 0.0352 & \\
\hline $\mathrm{H} 12 \mathrm{~A}$ & 0.3440 & 0.7461 & 0.7427 & $0.042 *$ & \\
\hline H12B & 0.4893 & 0.7156 & 0.7791 & $0.042 *$ & \\
\hline $\mathrm{C} 13$ & $0.40305(17)$ & $0.57970(12)$ & $0.73084(7)$ & $0.0336(3)$ & \\
\hline C14 & $0.24614(18)$ & $0.53463(12)$ & $0.71963(7)$ & $0.0340(3)$ & \\
\hline H14 & 0.1906 & 0.5951 & 0.6989 & $0.041^{*}$ & \\
\hline $\mathrm{C} 15$ & $0.2669(2)$ & $0.44163(14)$ & $0.67029(8)$ & $0.0438(4)$ & \\
\hline $\mathrm{H} 15 \mathrm{~A}$ & 0.1773 & 0.4307 & 0.6459 & $0.053^{*}$ & \\
\hline H15B & 0.2934 & 0.3710 & 0.6903 & $0.053^{*}$ & \\
\hline $\mathrm{C} 16$ & $0.3930(2)$ & $0.48456(16)$ & $0.62797(8)$ & $0.0483(4)$ & \\
\hline H16A & 0.3556 & 0.5039 & 0.5864 & $0.058 *$ & \\
\hline H16B & 0.4679 & 0.4268 & 0.6232 & $0.058^{*}$ & \\
\hline $\mathrm{C} 17$ & $0.4592(2)$ & $0.58969(13)$ & $0.66039(7)$ & 0.0388 & \\
\hline $\mathrm{C} 18$ & $0.1214(2)$ & $0.52665(18)$ & $0.93282(8)$ & 0.0505 & \\
\hline $\mathrm{H} 18 \mathrm{~A}$ & 0.0463 & 0.5073 & 0.9630 & $0.076^{*}$ & \\
\hline H18B & 0.2007 & 0.5648 & 0.9540 & $0.076^{*}$ & \\
\hline $\mathrm{H} 18 \mathrm{C}$ & 0.1583 & 0.4593 & 0.9133 & $0.076^{*}$ & \\
\hline C19 & $0.5017(2)$ & $0.49307(15)$ & $0.76391(8)$ & $0.0423(4)$ & \\
\hline H19A & 0.5989 & 0.5239 & 0.7693 & $0.063 *$ & \\
\hline H19B & 0.5073 & 0.4261 & 0.7386 & $0.063^{*}$ & \\
\hline H19C & 0.4607 & 0.4750 & 0.8045 & $0.063^{*}$ & \\
\hline $\mathrm{C} 20$ & $0.6275(2)$ & $0.59745(15)$ & $0.65638(8)$ & $0.0460(4)$ & \\
\hline $\mathrm{C} 21$ & $0.7000(3)$ & $0.7106(2)$ & $0.66925(12)$ & $0.0609(5)$ & \\
\hline $\mathrm{H} 21 \mathrm{~A}$ & $0.672(3)$ & $0.740(3)$ & $0.7116(14)$ & $0.076(8)^{*}$ & \\
\hline $\mathrm{H} 21 \mathrm{~B}$ & $0.656(4)$ & $0.773(3)$ & $0.6395(17)$ & $0.100(11)^{*}$ & \\
\hline $\mathrm{C} 22$ & $0.4291(3)$ & $0.71949(17)$ & $0.57472(9)$ & $0.0525(5)$ & \\
\hline $\mathrm{C} 23$ & $0.3459(4)$ & $0.8215(2)$ & $0.55363(12)$ & $0.0835(8)$ & \\
\hline $\mathrm{H} 23 \mathrm{~A}$ & 0.2414 & 0.8036 & 0.5535 & $0.100 *$ & \\
\hline $\mathrm{H} 23 \mathrm{~B}$ & 0.3612 & 0.8807 & 0.5846 & $0.100 *$ & \\
\hline $\mathrm{C} 24$ & $0.3861(5)$ & $0.8661(3)$ & $0.49010(13)$ & $0.0950(10)$ & \\
\hline $\mathrm{H} 24 \mathrm{~A}$ & 0.3902 & 0.8041 & 0.4602 & $0.114^{*}$ & $0.742(6)$ \\
\hline H24B & 0.3098 & 0.9176 & 0.4760 & $0.114^{*}$ & $0.742(6)$ \\
\hline $\mathrm{H} 24 \mathrm{C}$ & 0.4756 & 0.8270 & 0.4776 & $0.114^{*}$ & $0.258(6)$ \\
\hline H24D & 0.3093 & 0.8410 & 0.4614 & $0.114^{*}$ & $0.258(6)$ \\
\hline $\mathrm{C} 25$ & $0.5263(6)$ & $0.9239(4)$ & $0.4900(2)$ & $0.1017(15)$ & $0.742(6)$ \\
\hline $\mathrm{H} 25 \mathrm{~A}$ & 0.5190 & 0.9910 & 0.5151 & $0.153 *$ & $0.742(6)$ \\
\hline
\end{tabular}




\begin{tabular}{llllll}
$\mathrm{H} 25 \mathrm{~B}$ & 0.5522 & 0.9436 & 0.4474 & $0.153^{*}$ & $0.742(6)$ \\
$\mathrm{H} 25 \mathrm{C}$ & 0.6008 & 0.8755 & 0.5074 & $0.153^{*}$ & $0.742(6)$ \\
$\mathrm{C} 25 \mathrm{~A}$ & $0.4109(19)$ & $0.9858(11)$ & $0.4760(7)$ & $0.1017(15)$ & $0.258(6)$ \\
$\mathrm{H} 25 \mathrm{D}$ & 0.3263 & 1.0159 & 0.4545 & $0.153^{*}$ & $0.258(6)$ \\
$\mathrm{H} 25 \mathrm{E}$ & 0.4965 & 0.9935 & 0.4496 & $0.153^{*}$ & $0.258(6)$ \\
$\mathrm{H} 25 \mathrm{~F}$ & 0.4262 & 1.0263 & 0.5148 & $0.153^{*}$ & $0.258(6)$ \\
\hline
\end{tabular}

Atomic displacement parameters $\left(\AA^{2}\right)$

\begin{tabular}{|c|c|c|c|c|c|c|}
\hline & $U^{11}$ & $U^{22}$ & $U^{33}$ & $U^{12}$ & $U^{13}$ & $U^{23}$ \\
\hline $\mathrm{O} 1$ & $0.0363(6)$ & $0.0593(7)$ & $0.0543(7)$ & $0.0030(5)$ & $0.0045(5)$ & $-0.0085(6)$ \\
\hline $\mathrm{O} 2$ & $0.0376(6)$ & $0.0639(8)$ & $0.0387(6)$ & $-0.0106(6)$ & $-0.0084(5)$ & $0.0032(5)$ \\
\hline $\mathrm{O} 3$ & $0.0531(8)$ & $0.0558(8)$ & $0.0784(10)$ & $0.0067(6)$ & $0.0191(7)$ & $-0.0034(7)$ \\
\hline $\mathrm{O} 4$ & $0.0565(9)$ & $0.0848(13)$ & $0.0961(14)$ & $-0.0210(9)$ & $0.0018(9)$ & $-0.0035(11)$ \\
\hline O5 & $0.0565(7)$ & $0.0433(6)$ & $0.0346(5)$ & $0.0032(5)$ & $0.0059(5)$ & $0.0017(5)$ \\
\hline O6 & $0.0899(12)$ & 0.0657 (9) & $0.0454(7)$ & $-0.0036(8)$ & $0.0209(8)$ & $-0.0014(7)$ \\
\hline $\mathrm{C} 1$ & $0.0325(7)$ & $0.0491(9)$ & $0.0411(8)$ & $-0.0043(6)$ & $0.0009(6)$ & $-0.0120(7)$ \\
\hline $\mathrm{C} 2$ & $0.0396(9)$ & $0.0630(11)$ & $0.0424(8)$ & $-0.0048(8)$ & $0.0017(7)$ & $-0.0162(8)$ \\
\hline C3 & $0.0339(8)$ & $0.0467(9)$ & $0.0367(7)$ & $-0.0017(6)$ & $0.0030(6)$ & $0.0003(6)$ \\
\hline $\mathrm{C} 4$ & $0.0315(7)$ & $0.0467(8)$ & $0.0443(8)$ & $-0.0054(7)$ & $-0.0030(6)$ & $-0.0040(7)$ \\
\hline $\mathrm{C} 5$ & $0.0348(8)$ & $0.0376(7)$ & $0.0395(7)$ & $-0.0034(6)$ & $-0.0011(6)$ & $0.0012(6)$ \\
\hline C6 & $0.0357(8)$ & $0.0385(8)$ & $0.0618(10)$ & $-0.0092(7)$ & $0.0071(7)$ & $-0.0071(8)$ \\
\hline C7 & $0.0400(9)$ & $0.0414(8)$ & $0.0505(9)$ & $-0.0077(7)$ & $-0.0009(7)$ & $-0.0115(7)$ \\
\hline $\mathrm{C} 8$ & $0.0341(7)$ & $0.0305(6)$ & $0.0363(7)$ & $-0.0022(6)$ & $0.0002(6)$ & $-0.0014(6)$ \\
\hline C9 & $0.0317(7)$ & $0.0300(6)$ & $0.0317(6)$ & $0.0007(5)$ & $-0.0030(5)$ & $-0.0010(5)$ \\
\hline $\mathrm{C} 10$ & $0.0288(7)$ & $0.0407(8)$ & $0.0324(7)$ & $-0.0001(6)$ & -0.0025 & $0.0013(6)$ \\
\hline C11 & $0.0371(8)$ & $0.0354(7)$ & $0.0340(7)$ & $-0.0057(6)$ & $0.0000(6)$ & $-0.0054(6)$ \\
\hline $\mathrm{C} 12$ & $0.0389(8)$ & $0.0298(7)$ & $0.0369(7)$ & $-0.0061(6)$ & $0.0028(6)$ & $-0.0044(6)$ \\
\hline $\mathrm{C} 13$ & $0.0364(7)$ & $0.0309(7)$ & $0.0336(7)$ & $-0.0027(6)$ & $0.0019(6)$ & $-0.0031(6)$ \\
\hline $\mathrm{C} 14$ & $0.0386(8)$ & $0.0302(7)$ & $0.0332(7)$ & $-0.0023(6)$ & $-0.0029(6)$ & $-0.0021(5)$ \\
\hline $\mathrm{C} 15$ & $0.0508(9)$ & $0.0395(8)$ & $0.0410(8)$ & $-0.0078(7)$ & $0.0012(7)$ & $-0.0106(7)$ \\
\hline $\mathrm{C} 16$ & $0.0569(10)$ & $0.0461(9)$ & $0.0418(8)$ & $-0.0085(8)$ & $0.0066(8)$ & $-0.0141(7)$ \\
\hline $\mathrm{C} 17$ & $0.0472(9)$ & $0.0338(7)$ & $0.0353(7)$ & $-0.0016(7)$ & $0.0050(6)$ & $-0.0047(6)$ \\
\hline $\mathrm{C} 18$ & $0.0401(9)$ & $0.0691(12)$ & $0.0422(8)$ & $0.0010(8)$ & $-0.0015(7)$ & $0.0187(8)$ \\
\hline C19 & $0.0439(9)$ & $0.0392(8)$ & $0.0438(8)$ & $0.0031(7)$ & $-0.0041(7)$ & $-0.0021(7)$ \\
\hline $\mathrm{C} 20$ & $0.0509(10)$ & $0.0455(9)$ & $0.0417(8)$ & $-0.0044(8)$ & $0.0107(7)$ & $-0.0035(7)$ \\
\hline $\mathrm{C} 21$ & $0.0530(11)$ & $0.0623(12)$ & $0.0675(13)$ & $-0.0153(10)$ & $0.0141(10)$ & $-0.0139(10)$ \\
\hline $\mathrm{C} 22$ & $0.0687(12)$ & $0.0512(10)$ & $0.0375(8)$ & $-0.0054(9)$ & $0.0054(8)$ & $-0.0015(7)$ \\
\hline $\mathrm{C} 23$ & $0.123(2)$ & $0.0740(15)$ & $0.0538(12)$ & $0.0197(16)$ & $0.0049(14)$ & $0.0188(11)$ \\
\hline $\mathrm{C} 24$ & $0.146(3)$ & $0.0853(19)$ & $0.0542(13)$ & $-0.007(2)$ & $-0.0056(17)$ & $0.0197(12)$ \\
\hline $\mathrm{C} 25$ & $0.112(4)$ & $0.098(3)$ & $0.096(3)$ & $-0.031(3)$ & -0.003 & $0.017(2)$ \\
\hline $\mathrm{C} 25 \mathrm{~A}$ & $0.112(4)$ & $0.098(3)$ & $0.096(3)$ & $-0.031(3)$ & $-0.003(3)$ & $0.017(2)$ \\
\hline
\end{tabular}

Geometric parameters $\left(\AA,{ }^{\circ}\right)$

\begin{tabular}{llll}
\hline $\mathrm{O} 1-\mathrm{C} 3$ & $1.223(2)$ & $\mathrm{C} 12-\mathrm{C} 13$ & $1.5332(19)$ \\
$\mathrm{O} 2-\mathrm{H} 2$ & 0.8200 & $\mathrm{C} 13-\mathrm{C} 14$ & $1.537(2)$ \\
$\mathrm{O} 2-\mathrm{C} 11$ & $1.426(2)$ & $\mathrm{C} 13-\mathrm{C} 17$ & $1.578(2)$
\end{tabular}




\begin{tabular}{|c|c|c|c|}
\hline $\mathrm{O} 3-\mathrm{C} 20$ & $1.202(2)$ & $\mathrm{C} 13-\mathrm{C} 19$ & $1.531(2)$ \\
\hline $\mathrm{O} 4-\mathrm{H} 4$ & $0.83(3)$ & $\mathrm{C} 14-\mathrm{H} 14$ & 0.9800 \\
\hline $\mathrm{O} 4-\mathrm{C} 21$ & $1.391(3)$ & $\mathrm{C} 14-\mathrm{C} 15$ & $1.531(2)$ \\
\hline $\mathrm{O} 5-\mathrm{C} 17$ & $1.454(2)$ & $\mathrm{C} 15-\mathrm{H} 15 \mathrm{~A}$ & 0.9700 \\
\hline $\mathrm{O} 5-\mathrm{C} 22$ & $1.353(2)$ & $\mathrm{C} 15-\mathrm{H} 15 \mathrm{~B}$ & 0.9700 \\
\hline $\mathrm{O} 6-\mathrm{C} 22$ & $1.199(3)$ & $\mathrm{C} 15-\mathrm{C} 16$ & $1.538(3)$ \\
\hline $\mathrm{C} 1-\mathrm{H} 1 \mathrm{~A}$ & 0.9700 & $\mathrm{C} 16-\mathrm{H} 16 \mathrm{~A}$ & 0.9700 \\
\hline $\mathrm{C} 1-\mathrm{H} 1 \mathrm{~B}$ & 0.9700 & $\mathrm{C} 16-\mathrm{H} 16 \mathrm{~B}$ & 0.9700 \\
\hline $\mathrm{C} 1-\mathrm{C} 2$ & $1.528(2)$ & $\mathrm{C} 16-\mathrm{C} 17$ & $1.545(2)$ \\
\hline $\mathrm{C} 1-\mathrm{C} 10$ & $1.543(2)$ & $\mathrm{C} 17-\mathrm{C} 20$ & $1.530(3)$ \\
\hline $\mathrm{C} 2-\mathrm{H} 2 \mathrm{~A}$ & 0.9700 & $\mathrm{C} 18-\mathrm{H} 18 \mathrm{~A}$ & 0.9600 \\
\hline $\mathrm{C} 2-\mathrm{H} 2 \mathrm{~B}$ & 0.9700 & $\mathrm{C} 18-\mathrm{H} 18 \mathrm{~B}$ & 0.9600 \\
\hline $\mathrm{C} 2-\mathrm{C} 3$ & $1.497(2)$ & $\mathrm{C} 18-\mathrm{H} 18 \mathrm{C}$ & 0.9600 \\
\hline $\mathrm{C} 3-\mathrm{C} 4$ & $1.457(2)$ & C19-H19A & 0.9600 \\
\hline $\mathrm{C} 4-\mathrm{H} 4 \mathrm{~A}$ & 0.9300 & C19-H19B & 0.9600 \\
\hline $\mathrm{C} 4-\mathrm{C} 5$ & $1.338(2)$ & $\mathrm{C} 19-\mathrm{H} 19 \mathrm{C}$ & 0.9600 \\
\hline $\mathrm{C} 5-\mathrm{C} 6$ & $1.502(2)$ & $\mathrm{C} 20-\mathrm{C} 21$ & $1.520(3)$ \\
\hline $\mathrm{C} 5-\mathrm{C} 10$ & $1.521(2)$ & $\mathrm{C} 21-\mathrm{H} 21 \mathrm{~A}$ & $1.00(3)$ \\
\hline C6-H6A & 0.9700 & $\mathrm{C} 21-\mathrm{H} 21 \mathrm{~B}$ & $1.05(4)$ \\
\hline C6-H6B & 0.9700 & $\mathrm{C} 22-\mathrm{C} 23$ & $1.495(3)$ \\
\hline $\mathrm{C} 6-\mathrm{C} 7$ & $1.521(3)$ & $\mathrm{C} 23-\mathrm{H} 23 \mathrm{~A}$ & 0.9700 \\
\hline C7-H7A & 0.9700 & $\mathrm{C} 23-\mathrm{H} 23 \mathrm{~B}$ & 0.9700 \\
\hline C7-H7B & 0.9700 & $\mathrm{C} 23-\mathrm{C} 24$ & $1.488(4)$ \\
\hline $\mathrm{C} 7-\mathrm{C} 8$ & $1.528(2)$ & $\mathrm{C} 24-\mathrm{H} 24 \mathrm{~A}$ & 0.9700 \\
\hline $\mathrm{C} 8-\mathrm{H} 8$ & 0.9800 & $\mathrm{C} 24-\mathrm{H} 24 \mathrm{~B}$ & 0.9700 \\
\hline $\mathrm{C} 8-\mathrm{C} 9$ & $1.5473(19)$ & $\mathrm{C} 24-\mathrm{H} 24 \mathrm{C}$ & 0.9700 \\
\hline $\mathrm{C} 8-\mathrm{C} 14$ & $1.527(2)$ & $\mathrm{C} 24-\mathrm{H} 24 \mathrm{D}$ & 0.9700 \\
\hline $\mathrm{C} 9-\mathrm{H} 9$ & 0.9800 & $\mathrm{C} 24-\mathrm{C} 25$ & $1.443(6)$ \\
\hline $\mathrm{C} 9-\mathrm{C} 10$ & $1.569(2)$ & $\mathrm{C} 24-\mathrm{C} 25 \mathrm{~A}$ & $1.470(12)$ \\
\hline $\mathrm{C} 9-\mathrm{C} 11$ & $1.547(2)$ & $\mathrm{C} 25-\mathrm{H} 25 \mathrm{~A}$ & 0.9600 \\
\hline $\mathrm{C} 10-\mathrm{C} 18$ & $1.550(2)$ & $\mathrm{C} 25-\mathrm{H} 25 \mathrm{~B}$ & 0.9600 \\
\hline C11-H11 & 0.9800 & $\mathrm{C} 25-\mathrm{H} 25 \mathrm{C}$ & 0.9600 \\
\hline $\mathrm{C} 11-\mathrm{C} 12$ & $1.545(2)$ & $\mathrm{C} 25 \mathrm{~A}-\mathrm{H} 25 \mathrm{D}$ & 0.9600 \\
\hline $\mathrm{C} 12-\mathrm{H} 12 \mathrm{~A}$ & 0.9700 & $\mathrm{C} 25 \mathrm{~A}-\mathrm{H} 25 \mathrm{E}$ & 0.9600 \\
\hline $\mathrm{C} 12-\mathrm{H} 12 \mathrm{~B}$ & 0.9700 & $\mathrm{C} 25 \mathrm{~A}-\mathrm{H} 25 \mathrm{~F}$ & 0.9600 \\
\hline $\mathrm{C} 11-\mathrm{O} 2-\mathrm{H} 2$ & 109.5 & $\mathrm{C} 14-\mathrm{C} 15-\mathrm{H} 15 \mathrm{~A}$ & 110.9 \\
\hline $\mathrm{C} 21-\mathrm{O} 4-\mathrm{H} 4$ & $101(2)$ & $\mathrm{C} 14-\mathrm{C} 15-\mathrm{H} 15 \mathrm{~B}$ & 110.9 \\
\hline $\mathrm{C} 22-\mathrm{O} 5-\mathrm{C} 17$ & $116.96(14)$ & $\mathrm{C} 14-\mathrm{C} 15-\mathrm{C} 16$ & $104.36(13)$ \\
\hline $\mathrm{H} 1 \mathrm{~A}-\mathrm{C} 1-\mathrm{H} 1 \mathrm{~B}$ & 107.7 & $\mathrm{H} 15 \mathrm{~A}-\mathrm{C} 15-\mathrm{H} 15 \mathrm{~B}$ & 108.9 \\
\hline $\mathrm{C} 2-\mathrm{C} 1-\mathrm{H} 1 \mathrm{~A}$ & 108.8 & $\mathrm{C} 16-\mathrm{C} 15-\mathrm{H} 15 \mathrm{~A}$ & 110.9 \\
\hline $\mathrm{C} 2-\mathrm{C} 1-\mathrm{H} 1 \mathrm{~B}$ & 108.8 & $\mathrm{C} 16-\mathrm{C} 15-\mathrm{H} 15 \mathrm{~B}$ & 110.9 \\
\hline $\mathrm{C} 2-\mathrm{C} 1-\mathrm{C} 10$ & $113.88(15)$ & $\mathrm{C} 15-\mathrm{C} 16-\mathrm{H} 16 \mathrm{~A}$ & 110.2 \\
\hline $\mathrm{C} 10-\mathrm{C} 1-\mathrm{H} 1 \mathrm{~A}$ & 108.8 & $\mathrm{C} 15-\mathrm{C} 16-\mathrm{H} 16 \mathrm{~B}$ & 110.2 \\
\hline $\mathrm{C} 10-\mathrm{C} 1-\mathrm{H} 1 \mathrm{~B}$ & 108.8 & $\mathrm{C} 15-\mathrm{C} 16-\mathrm{C} 17$ & $107.35(13)$ \\
\hline $\mathrm{C} 1-\mathrm{C} 2-\mathrm{H} 2 \mathrm{~A}$ & 109.5 & $\mathrm{H} 16 \mathrm{~A}-\mathrm{C} 16-\mathrm{H} 16 \mathrm{~B}$ & 108.5 \\
\hline $\mathrm{C} 1-\mathrm{C} 2-\mathrm{H} 2 \mathrm{~B}$ & 109.5 & $\mathrm{C} 17-\mathrm{C} 16-\mathrm{H} 16 \mathrm{~A}$ & 110.2 \\
\hline $\mathrm{H} 2 \mathrm{~A}-\mathrm{C} 2-\mathrm{H} 2 \mathrm{~B}$ & 108.1 & $\mathrm{C} 17-\mathrm{C} 16-\mathrm{H} 16 \mathrm{~B}$ & 110.2 \\
\hline
\end{tabular}




\begin{tabular}{|c|c|c|c|}
\hline $\mathrm{C} 3-\mathrm{C} 2-\mathrm{C} 1$ & $110.66(13)$ & $\mathrm{O} 5-\mathrm{C} 17-\mathrm{C} 13$ & $106.28(12)$ \\
\hline $\mathrm{C} 3-\mathrm{C} 2-\mathrm{H} 2 \mathrm{~A}$ & 109.5 & $\mathrm{O} 5-\mathrm{C} 17-\mathrm{C} 16$ & $111.11(14)$ \\
\hline $\mathrm{C} 3-\mathrm{C} 2-\mathrm{H} 2 \mathrm{~B}$ & 109.5 & $\mathrm{O} 5-\mathrm{C} 17-\mathrm{C} 20$ & $109.19(14)$ \\
\hline $\mathrm{O} 1-\mathrm{C} 3-\mathrm{C} 2$ & $122.63(15)$ & $\mathrm{C} 16-\mathrm{C} 17-\mathrm{C} 13$ & $103.46(13)$ \\
\hline $\mathrm{O} 1-\mathrm{C} 3-\mathrm{C} 4$ & $121.47(15)$ & $\mathrm{C} 20-\mathrm{C} 17-\mathrm{C} 13$ & $112.19(14)$ \\
\hline $\mathrm{C} 4-\mathrm{C} 3-\mathrm{C} 2$ & $115.82(15)$ & $\mathrm{C} 20-\mathrm{C} 17-\mathrm{C} 16$ & $114.24(14)$ \\
\hline $\mathrm{C} 3-\mathrm{C} 4-\mathrm{H} 4 \mathrm{~A}$ & 118.0 & $\mathrm{C} 10-\mathrm{C} 18-\mathrm{H} 18 \mathrm{~A}$ & 109.5 \\
\hline $\mathrm{C} 5-\mathrm{C} 4-\mathrm{C} 3$ & $124.03(15)$ & $\mathrm{C} 10-\mathrm{C} 18-\mathrm{H} 18 \mathrm{~B}$ & 109.5 \\
\hline $\mathrm{C} 5-\mathrm{C} 4-\mathrm{H} 4 \mathrm{~A}$ & 118.0 & $\mathrm{C} 10-\mathrm{C} 18-\mathrm{H} 18 \mathrm{C}$ & 109.5 \\
\hline $\mathrm{C} 4-\mathrm{C} 5-\mathrm{C} 6$ & $120.77(15)$ & $\mathrm{H} 18 \mathrm{~A}-\mathrm{C} 18-\mathrm{H} 18 \mathrm{~B}$ & 109.5 \\
\hline $\mathrm{C} 4-\mathrm{C} 5-\mathrm{C} 10$ & $123.13(14)$ & $\mathrm{H} 18 \mathrm{~A}-\mathrm{C} 18-\mathrm{H} 18 \mathrm{C}$ & 109.5 \\
\hline $\mathrm{C} 6-\mathrm{C} 5-\mathrm{C} 10$ & $116.07(14)$ & $\mathrm{H} 18 \mathrm{~B}-\mathrm{C} 18-\mathrm{H} 18 \mathrm{C}$ & 109.5 \\
\hline $\mathrm{C} 5-\mathrm{C} 6-\mathrm{H} 6 \mathrm{~A}$ & 109.3 & $\mathrm{C} 13-\mathrm{C} 19-\mathrm{H} 19 \mathrm{~A}$ & 109.5 \\
\hline $\mathrm{C} 5-\mathrm{C} 6-\mathrm{H} 6 \mathrm{~B}$ & 109.3 & $\mathrm{C} 13-\mathrm{C} 19-\mathrm{H} 19 \mathrm{~B}$ & 109.5 \\
\hline $\mathrm{C} 5-\mathrm{C} 6-\mathrm{C} 7$ & $111.82(14)$ & $\mathrm{C} 13-\mathrm{C} 19-\mathrm{H} 19 \mathrm{C}$ & 109.5 \\
\hline $\mathrm{H} 6 \mathrm{~A}-\mathrm{C} 6-\mathrm{H} 6 \mathrm{~B}$ & 107.9 & $\mathrm{H} 19 \mathrm{~A}-\mathrm{C} 19-\mathrm{H} 19 \mathrm{~B}$ & 109.5 \\
\hline $\mathrm{C} 7-\mathrm{C} 6-\mathrm{H} 6 \mathrm{~A}$ & 109.3 & $\mathrm{H} 19 \mathrm{~A}-\mathrm{C} 19-\mathrm{H} 19 \mathrm{C}$ & 109.5 \\
\hline $\mathrm{C} 7-\mathrm{C} 6-\mathrm{H} 6 \mathrm{~B}$ & 109.3 & $\mathrm{H} 19 \mathrm{~B}-\mathrm{C} 19-\mathrm{H} 19 \mathrm{C}$ & 109.5 \\
\hline $\mathrm{C} 6-\mathrm{C} 7-\mathrm{H} 7 \mathrm{~A}$ & 109.1 & $\mathrm{O} 3-\mathrm{C} 20-\mathrm{C} 17$ & $122.78(17)$ \\
\hline $\mathrm{C} 6-\mathrm{C} 7-\mathrm{H} 7 \mathrm{~B}$ & 109.1 & $\mathrm{O} 3-\mathrm{C} 20-\mathrm{C} 21$ & $118.72(18)$ \\
\hline $\mathrm{C} 6-\mathrm{C} 7-\mathrm{C} 8$ & $112.56(14)$ & $\mathrm{C} 21-\mathrm{C} 20-\mathrm{C} 17$ & $118.30(16)$ \\
\hline $\mathrm{H} 7 \mathrm{~A}-\mathrm{C} 7-\mathrm{H} 7 \mathrm{~B}$ & 107.8 & $\mathrm{O} 4-\mathrm{C} 21-\mathrm{C} 20$ & $112.0(2)$ \\
\hline $\mathrm{C} 8-\mathrm{C} 7-\mathrm{H} 7 \mathrm{~A}$ & 109.1 & $\mathrm{O} 4-\mathrm{C} 21-\mathrm{H} 21 \mathrm{~A}$ & $105.3(18)$ \\
\hline $\mathrm{C} 8-\mathrm{C} 7-\mathrm{H} 7 \mathrm{~B}$ & 109.1 & $\mathrm{O} 4-\mathrm{C} 21-\mathrm{H} 21 \mathrm{~B}$ & $115.8(19)$ \\
\hline $\mathrm{C} 7-\mathrm{C} 8-\mathrm{H} 8$ & 109.3 & $\mathrm{C} 20-\mathrm{C} 21-\mathrm{H} 21 \mathrm{~A}$ & $111.5(17)$ \\
\hline $\mathrm{C} 7-\mathrm{C} 8-\mathrm{C} 9$ & $110.74(13)$ & $\mathrm{C} 20-\mathrm{C} 21-\mathrm{H} 21 \mathrm{~B}$ & $111(2)$ \\
\hline $\mathrm{C} 9-\mathrm{C} 8-\mathrm{H} 8$ & 109.3 & $\mathrm{H} 21 \mathrm{~A}-\mathrm{C} 21-\mathrm{H} 21 \mathrm{~B}$ & $101(3)$ \\
\hline $\mathrm{C} 14-\mathrm{C} 8-\mathrm{C} 7$ & $109.98(13)$ & $\mathrm{O} 5-\mathrm{C} 22-\mathrm{C} 23$ & $110.96(18)$ \\
\hline $\mathrm{C} 14-\mathrm{C} 8-\mathrm{H} 8$ & 109.3 & $\mathrm{O} 6-\mathrm{C} 22-\mathrm{O} 5$ & $122.50(19)$ \\
\hline $\mathrm{C} 14-\mathrm{C} 8-\mathrm{C} 9$ & $108.24(11)$ & $\mathrm{O} 6-\mathrm{C} 22-\mathrm{C} 23$ & $126.5(2)$ \\
\hline $\mathrm{C} 8-\mathrm{C} 9-\mathrm{H} 9$ & 104.7 & $\mathrm{C} 22-\mathrm{C} 23-\mathrm{H} 23 \mathrm{~A}$ & 108.4 \\
\hline $\mathrm{C} 8-\mathrm{C} 9-\mathrm{C} 10$ & $114.20(12)$ & $\mathrm{C} 22-\mathrm{C} 23-\mathrm{H} 23 \mathrm{~B}$ & 108.4 \\
\hline $\mathrm{C} 10-\mathrm{C} 9-\mathrm{H} 9$ & 104.7 & $\mathrm{H} 23 \mathrm{~A}-\mathrm{C} 23-\mathrm{H} 23 \mathrm{~B}$ & 107.4 \\
\hline $\mathrm{C} 11-\mathrm{C} 9-\mathrm{C} 8$ & $113.00(12)$ & $\mathrm{C} 24-\mathrm{C} 23-\mathrm{C} 22$ & $115.7(3)$ \\
\hline $\mathrm{C} 11-\mathrm{C} 9-\mathrm{H} 9$ & 104.7 & $\mathrm{C} 24-\mathrm{C} 23-\mathrm{H} 23 \mathrm{~A}$ & 108.4 \\
\hline $\mathrm{C} 11-\mathrm{C} 9-\mathrm{C} 10$ & $114.29(12)$ & $\mathrm{C} 24-\mathrm{C} 23-\mathrm{H} 23 \mathrm{~B}$ & 108.4 \\
\hline $\mathrm{C} 1-\mathrm{C} 10-\mathrm{C} 9$ & $108.16(12)$ & $\mathrm{C} 23-\mathrm{C} 24-\mathrm{H} 24 \mathrm{~A}$ & 109.0 \\
\hline $\mathrm{C} 1-\mathrm{C} 10-\mathrm{C} 18$ & $110.12(14)$ & $\mathrm{C} 23-\mathrm{C} 24-\mathrm{H} 24 \mathrm{~B}$ & 109.0 \\
\hline $\mathrm{C} 5-\mathrm{C} 10-\mathrm{C} 1$ & $110.03(13)$ & $\mathrm{C} 23-\mathrm{C} 24-\mathrm{H} 24 \mathrm{C}$ & 106.2 \\
\hline $\mathrm{C} 5-\mathrm{C} 10-\mathrm{C} 9$ & $107.82(12)$ & $\mathrm{C} 23-\mathrm{C} 24-\mathrm{H} 24 \mathrm{D}$ & 106.2 \\
\hline $\mathrm{C} 5-\mathrm{C} 10-\mathrm{C} 18$ & $106.05(13)$ & $\mathrm{H} 24 \mathrm{~A}-\mathrm{C} 24-\mathrm{H} 24 \mathrm{~B}$ & 107.8 \\
\hline $\mathrm{C} 18-\mathrm{C} 10-\mathrm{C} 9$ & $114.58(13)$ & $\mathrm{H} 24 \mathrm{~A}-\mathrm{C} 24-\mathrm{H} 24 \mathrm{C}$ & 55.2 \\
\hline $\mathrm{O} 2-\mathrm{C} 11-\mathrm{C} 9$ & $109.66(12)$ & $\mathrm{H} 24 \mathrm{~A}-\mathrm{C} 24-\mathrm{H} 24 \mathrm{D}$ & 52.2 \\
\hline $\mathrm{O} 2-\mathrm{C} 11-\mathrm{H} 11$ & 107.6 & $\mathrm{H} 24 \mathrm{~B}-\mathrm{C} 24-\mathrm{H} 24 \mathrm{C}$ & 144.4 \\
\hline $\mathrm{O} 2-\mathrm{C} 11-\mathrm{C} 12$ & $111.49(13)$ & $\mathrm{H} 24 \mathrm{~B}-\mathrm{C} 24-\mathrm{H} 24 \mathrm{D}$ & 59.4 \\
\hline $\mathrm{C} 9-\mathrm{C} 11-\mathrm{H} 11$ & 107.6 & $\mathrm{H} 24 \mathrm{C}-\mathrm{C} 24-\mathrm{H} 24 \mathrm{D}$ & 106.4 \\
\hline $\mathrm{C} 12-\mathrm{C} 11-\mathrm{C} 9$ & $112.59(12)$ & $\mathrm{C} 25-\mathrm{C} 24-\mathrm{C} 23$ & $112.7(3)$ \\
\hline $\mathrm{C} 12-\mathrm{C} 11-\mathrm{H} 11$ & 107.6 & $\mathrm{C} 25-\mathrm{C} 24-\mathrm{H} 24 \mathrm{~A}$ & 109.0 \\
\hline
\end{tabular}




\begin{tabular}{|c|c|c|c|}
\hline $\mathrm{C} 11-\mathrm{C} 12-\mathrm{H} 12 \mathrm{~A}$ & 108.9 & $\mathrm{C} 25-\mathrm{C} 24-\mathrm{H} 24 \mathrm{~B}$ & 109.0 \\
\hline $\mathrm{C} 11-\mathrm{C} 12-\mathrm{H} 12 \mathrm{~B}$ & 108.9 & $\mathrm{C} 25-\mathrm{C} 24-\mathrm{H} 24 \mathrm{C}$ & 59.5 \\
\hline $\mathrm{H} 12 \mathrm{~A}-\mathrm{C} 12-\mathrm{H} 12 \mathrm{~B}$ & 107.7 & $\mathrm{C} 25-\mathrm{C} 24-\mathrm{H} 24 \mathrm{D}$ & 140.9 \\
\hline $\mathrm{C} 13-\mathrm{C} 12-\mathrm{C} 11$ & $113.29(12)$ & $\mathrm{C} 25-\mathrm{C} 24-\mathrm{C} 25 \mathrm{~A}$ & $53.5(7)$ \\
\hline $\mathrm{C} 13-\mathrm{C} 12-\mathrm{H} 12 \mathrm{~A}$ & 108.9 & $\mathrm{C} 25 \mathrm{~A}-\mathrm{C} 24-\mathrm{C} 23$ & $124.3(7)$ \\
\hline $\mathrm{C} 13-\mathrm{C} 12-\mathrm{H} 12 \mathrm{~B}$ & 108.9 & $\mathrm{C} 25 \mathrm{~A}-\mathrm{C} 24-\mathrm{H} 24 \mathrm{~A}$ & 126.6 \\
\hline $\mathrm{C} 12-\mathrm{C} 13-\mathrm{C} 14$ & $108.01(12)$ & $\mathrm{C} 25 \mathrm{~A}-\mathrm{C} 24-\mathrm{H} 24 \mathrm{~B}$ & 55.7 \\
\hline $\mathrm{C} 12-\mathrm{C} 13-\mathrm{C} 17$ & $117.02(12)$ & $\mathrm{C} 25 \mathrm{~A}-\mathrm{C} 24-\mathrm{H} 24 \mathrm{C}$ & 106.2 \\
\hline $\mathrm{C} 14-\mathrm{C} 13-\mathrm{C} 17$ & $100.28(12)$ & $\mathrm{C} 25 \mathrm{~A}-\mathrm{C} 24-\mathrm{H} 24 \mathrm{D}$ & 106.2 \\
\hline $\mathrm{C} 19-\mathrm{C} 13-\mathrm{C} 12$ & $111.91(13)$ & $\mathrm{C} 24-\mathrm{C} 25-\mathrm{H} 25 \mathrm{~A}$ & 109.5 \\
\hline $\mathrm{C} 19-\mathrm{C} 13-\mathrm{C} 14$ & $112.07(12)$ & $\mathrm{C} 24-\mathrm{C} 25-\mathrm{H} 25 \mathrm{~B}$ & 109.5 \\
\hline $\mathrm{C} 19-\mathrm{C} 13-\mathrm{C} 17$ & $107.06(13)$ & $\mathrm{C} 24-\mathrm{C} 25-\mathrm{H} 25 \mathrm{C}$ & 109.5 \\
\hline $\mathrm{C} 8-\mathrm{C} 14-\mathrm{C} 13$ & $112.92(12)$ & $\mathrm{C} 24-\mathrm{C} 25 \mathrm{~A}-\mathrm{H} 25 \mathrm{D}$ & 109.5 \\
\hline $\mathrm{C} 8-\mathrm{C} 14-\mathrm{H} 14$ & 106.7 & $\mathrm{C} 24-\mathrm{C} 25 \mathrm{~A}-\mathrm{H} 25 \mathrm{E}$ & 109.5 \\
\hline $\mathrm{C} 8-\mathrm{C} 14-\mathrm{C} 15$ & $118.96(13)$ & $\mathrm{C} 24-\mathrm{C} 25 \mathrm{~A}-\mathrm{H} 25 \mathrm{~F}$ & 109.5 \\
\hline $\mathrm{C} 13-\mathrm{C} 14-\mathrm{H} 14$ & 106.7 & $\mathrm{H} 25 \mathrm{D}-\mathrm{C} 25 \mathrm{~A}-\mathrm{H} 25 \mathrm{E}$ & 109.5 \\
\hline $\mathrm{C} 15-\mathrm{C} 14-\mathrm{C} 13$ & $104.06(13)$ & $\mathrm{H} 25 \mathrm{D}-\mathrm{C} 25 \mathrm{~A}-\mathrm{H} 25 \mathrm{~F}$ & 109.5 \\
\hline $\mathrm{C} 15-\mathrm{C} 14-\mathrm{H} 14$ & 106.7 & $\mathrm{H} 25 \mathrm{E}-\mathrm{C} 25 \mathrm{~A}-\mathrm{H} 25 \mathrm{~F}$ & 109.5 \\
\hline $\mathrm{O} 1-\mathrm{C} 3-\mathrm{C} 4-\mathrm{C} 5$ & $175.19(17)$ & $\mathrm{C} 10-\mathrm{C} 9-\mathrm{C} 11-\mathrm{C} 12$ & $-178.22(13)$ \\
\hline $\mathrm{O} 2-\mathrm{C} 11-\mathrm{C} 12-\mathrm{C} 13$ & $74.10(17)$ & $\mathrm{C} 11-\mathrm{C} 9-\mathrm{C} 10-\mathrm{C} 1$ & $57.46(16)$ \\
\hline $\mathrm{O} 3-\mathrm{C} 20-\mathrm{C} 21-\mathrm{O} 4$ & $-1.1(3)$ & $\mathrm{C} 11-\mathrm{C} 9-\mathrm{C} 10-\mathrm{C} 5$ & $176.41(12)$ \\
\hline $\mathrm{O} 5-\mathrm{C} 17-\mathrm{C} 20-\mathrm{O} 3$ & $-147.68(18)$ & $\mathrm{C} 11-\mathrm{C} 9-\mathrm{C} 10-\mathrm{C} 18$ & $-65.79(18)$ \\
\hline $\mathrm{O} 5-\mathrm{C} 17-\mathrm{C} 20-\mathrm{C} 21$ & $37.6(2)$ & $\mathrm{C} 11-\mathrm{C} 12-\mathrm{C} 13-\mathrm{C} 14$ & $54.22(17)$ \\
\hline $\mathrm{O} 5-\mathrm{C} 22-\mathrm{C} 23-\mathrm{C} 24$ & $174.0(3)$ & $\mathrm{C} 11-\mathrm{C} 12-\mathrm{C} 13-\mathrm{C} 17$ & $166.34(14)$ \\
\hline $\mathrm{O} 6-\mathrm{C} 22-\mathrm{C} 23-\mathrm{C} 24$ & $-5.4(5)$ & $\mathrm{C} 11-\mathrm{C} 12-\mathrm{C} 13-\mathrm{C} 19$ & $-69.61(18)$ \\
\hline $\mathrm{C} 1-\mathrm{C} 2-\mathrm{C} 3-\mathrm{O} 1$ & $-145.75(18)$ & $\mathrm{C} 12-\mathrm{C} 13-\mathrm{C} 14-\mathrm{C} 8$ & $-61.36(15)$ \\
\hline $\mathrm{C} 1-\mathrm{C} 2-\mathrm{C} 3-\mathrm{C} 4$ & $37.2(2)$ & $\mathrm{C} 12-\mathrm{C} 13-\mathrm{C} 14-\mathrm{C} 15$ & $168.27(12)$ \\
\hline $\mathrm{C} 2-\mathrm{C} 1-\mathrm{C} 10-\mathrm{C} 5$ & $42.57(18)$ & $\mathrm{C} 12-\mathrm{C} 13-\mathrm{C} 17-\mathrm{O} 5$ & $-37.73(19)$ \\
\hline $\mathrm{C} 2-\mathrm{C} 1-\mathrm{C} 10-\mathrm{C} 9$ & $160.11(14)$ & $\mathrm{C} 12-\mathrm{C} 13-\mathrm{C} 17-\mathrm{C} 16$ & $-154.84(15)$ \\
\hline $\mathrm{C} 2-\mathrm{C} 1-\mathrm{C} 10-\mathrm{C} 18$ & $-73.98(18)$ & $\mathrm{C} 12-\mathrm{C} 13-\mathrm{C} 17-\mathrm{C} 20$ & $81.54(18)$ \\
\hline $\mathrm{C} 2-\mathrm{C} 3-\mathrm{C} 4-\mathrm{C} 5$ & $-7.8(3)$ & $\mathrm{C} 13-\mathrm{C} 14-\mathrm{C} 15-\mathrm{C} 16$ & $-34.65(17)$ \\
\hline $\mathrm{C} 3-\mathrm{C} 4-\mathrm{C} 5-\mathrm{C} 6$ & $172.34(16)$ & $\mathrm{C} 13-\mathrm{C} 17-\mathrm{C} 20-\mathrm{O} 3$ & $94.8(2)$ \\
\hline $\mathrm{C} 3-\mathrm{C} 4-\mathrm{C} 5-\mathrm{C} 10$ & $-5.4(3)$ & $\mathrm{C} 13-\mathrm{C} 17-\mathrm{C} 20-\mathrm{C} 21$ & $-80.0(2)$ \\
\hline $\mathrm{C} 4-\mathrm{C} 5-\mathrm{C} 6-\mathrm{C} 7$ & $127.75(18)$ & $\mathrm{C} 14-\mathrm{C} 8-\mathrm{C} 9-\mathrm{C} 10$ & $173.70(12)$ \\
\hline $\mathrm{C} 4-\mathrm{C} 5-\mathrm{C} 10-\mathrm{C} 1$ & $-12.2(2)$ & $\mathrm{C} 14-\mathrm{C} 8-\mathrm{C} 9-\mathrm{C} 11$ & $-53.41(16)$ \\
\hline $\mathrm{C} 4-\mathrm{C} 5-\mathrm{C} 10-\mathrm{C} 9$ & $-130.00(16)$ & $\mathrm{C} 14-\mathrm{C} 13-\mathrm{C} 17-\mathrm{O} 5$ & $78.71(13)$ \\
\hline $\mathrm{C} 4-\mathrm{C} 5-\mathrm{C} 10-\mathrm{C} 18$ & $106.83(19)$ & $\mathrm{C} 14-\mathrm{C} 13-\mathrm{C} 17-\mathrm{C} 16$ & $-38.40(15)$ \\
\hline $\mathrm{C} 5-\mathrm{C} 6-\mathrm{C} 7-\mathrm{C} 8$ & $52.7(2)$ & $\mathrm{C} 14-\mathrm{C} 13-\mathrm{C} 17-\mathrm{C} 20$ & $-162.02(13)$ \\
\hline $\mathrm{C} 6-\mathrm{C} 5-\mathrm{C} 10-\mathrm{C} 1$ & $169.87(14)$ & $\mathrm{C} 14-\mathrm{C} 15-\mathrm{C} 16-\mathrm{C} 17$ & $9.6(2)$ \\
\hline $\mathrm{C} 6-\mathrm{C} 5-\mathrm{C} 10-\mathrm{C} 9$ & $52.12(18)$ & $\mathrm{C} 15-\mathrm{C} 16-\mathrm{C} 17-\mathrm{O} 5$ & $-95.58(17)$ \\
\hline $\mathrm{C} 6-\mathrm{C} 5-\mathrm{C} 10-\mathrm{C} 18$ & $-71.05(18)$ & $\mathrm{C} 15-\mathrm{C} 16-\mathrm{C} 17-\mathrm{C} 13$ & $18.09(19)$ \\
\hline $\mathrm{C} 6-\mathrm{C} 7-\mathrm{C} 8-\mathrm{C} 9$ & $-52.40(19)$ & $\mathrm{C} 15-\mathrm{C} 16-\mathrm{C} 17-\mathrm{C} 20$ & $140.35(17)$ \\
\hline $\mathrm{C} 6-\mathrm{C} 7-\mathrm{C} 8-\mathrm{C} 14$ & $-171.99(14)$ & $\mathrm{C} 16-\mathrm{C} 17-\mathrm{C} 20-\mathrm{O} 3$ & $-22.6(3)$ \\
\hline $\mathrm{C} 7-\mathrm{C} 8-\mathrm{C} 9-\mathrm{C} 10$ & $53.07(17)$ & $\mathrm{C} 16-\mathrm{C} 17-\mathrm{C} 20-\mathrm{C} 21$ & $162.70(18)$ \\
\hline $\mathrm{C} 7-\mathrm{C} 8-\mathrm{C} 9-\mathrm{C} 11$ & $-174.04(13)$ & $\mathrm{C} 17-\mathrm{O} 5-\mathrm{C} 22-\mathrm{O} 6$ & $-5.1(3)$ \\
\hline $\mathrm{C} 7-\mathrm{C} 8-\mathrm{C} 14-\mathrm{C} 13$ & $-177.98(13)$ & $\mathrm{C} 17-\mathrm{O} 5-\mathrm{C} 22-\mathrm{C} 23$ & $175.4(2)$ \\
\hline $\mathrm{C} 7-\mathrm{C} 8-\mathrm{C} 14-\mathrm{C} 15$ & $-55.61(19)$ & $\mathrm{C} 17-\mathrm{C} 13-\mathrm{C} 14-\mathrm{C} 8$ & $175.66(12)$ \\
\hline
\end{tabular}




\section{supporting information}

$\begin{array}{ll}\mathrm{C} 8-\mathrm{C} 9-\mathrm{C} 10-\mathrm{C} 1 & -170.26(12) \\ \mathrm{C} 8-\mathrm{C} 9-\mathrm{C} 10-\mathrm{C} 5 & -51.30(16) \\ \mathrm{C} 8-\mathrm{C} 9-\mathrm{C} 10-\mathrm{C} 18 & 66.49(17) \\ \mathrm{C} 8-\mathrm{C} 9-\mathrm{C} 11-\mathrm{O} 2 & -75.82(15) \\ \mathrm{C} 8-\mathrm{C} 9-\mathrm{C} 11-\mathrm{C} 12 & 48.93(17) \\ \mathrm{C} 8-\mathrm{C} 14-\mathrm{C} 15-\mathrm{C} 16 & -161.34(15) \\ \mathrm{C} 9-\mathrm{C} 8-\mathrm{C} 14-\mathrm{C} 13 & 60.92(15) \\ \mathrm{C} 9-\mathrm{C} 8-\mathrm{C} 14-\mathrm{C} 15 & -176.71(14) \\ \mathrm{C} 9-\mathrm{C} 11-\mathrm{C} 12-\mathrm{C} 13 & -49.64(18) \\ \mathrm{C} 10-\mathrm{C} 1-\mathrm{C} 2-\mathrm{C} 3 & -55.8(2) \\ \mathrm{C} 10-\mathrm{C} 5-\mathrm{C} 6-\mathrm{C} 7 & -54.3(2) \\ \mathrm{C} 10-\mathrm{C} 9-\mathrm{C} 11-\mathrm{O} 2 & 57.03(16)\end{array}$

$$
\begin{aligned}
& \mathrm{C} 17-\mathrm{C} 13-\mathrm{C} 14-\mathrm{C} 15 \\
& \mathrm{C} 17-\mathrm{C} 20-\mathrm{C} 21-\mathrm{O} 4 \\
& \mathrm{C} 19-\mathrm{C} 13-\mathrm{C} 14-\mathrm{C} 8 \\
& \mathrm{C} 19-\mathrm{C} 13-\mathrm{C} 14-\mathrm{C} 15 \\
& \mathrm{C} 19-\mathrm{C} 13-\mathrm{C} 17-\mathrm{O} 5 \\
& \mathrm{C} 19-\mathrm{C} 13-\mathrm{C} 17-\mathrm{C} 16 \\
& \mathrm{C} 19-\mathrm{C} 13-\mathrm{C} 17-\mathrm{C} 20 \\
& \mathrm{C} 22-\mathrm{O} 5-\mathrm{C} 17-\mathrm{C} 13 \\
& \mathrm{C} 22-\mathrm{O} 5-\mathrm{C} 17-\mathrm{C} 16 \\
& \mathrm{C} 22-\mathrm{O} 5-\mathrm{C} 17-\mathrm{C} 20 \\
& \mathrm{C} 22-\mathrm{C} 23-\mathrm{C} 24-\mathrm{C} 25 \\
& \mathrm{C} 22-\mathrm{C} 23-\mathrm{C} 24-\mathrm{C} 25 \mathrm{~A}
\end{aligned}
$$

45.28 (14)

$173.85(19)$

$62.37(16)$

$-68.01(16)$

$-164.22(13)$

78.68 (16)

$-44.94(17)$

$179.33(15)$

$-68.78(19)$

$58.11(19)$

-74.3 (4)

$-134.6(9)$

Hydrogen-bond geometry $\left(\AA,{ }^{\circ}\right)$

\begin{tabular}{lllll}
\hline$D-\mathrm{H} \cdots A$ & $D-\mathrm{H}$ & $\mathrm{H} \cdots A$ & $D \cdots A$ & $D-\mathrm{H} \cdots A$ \\
\hline $\mathrm{C} 19-\mathrm{H} 19 C \cdots \mathrm{O} 2$ & 0.96 & 2.39 & $3.016(2)$ & 122 \\
$\mathrm{O} 4-\mathrm{H} 4 \cdots \mathrm{O} 3$ & $0.83(3)$ & $2.06(3)$ & $2.629(3)$ & $126(2)$ \\
$\mathrm{O} 2-\mathrm{H} 2 \cdots \mathrm{O} 1{ }^{\mathrm{i}}$ & 0.82 & 2.11 & $2.9192(18)$ & 169 \\
\hline
\end{tabular}

Symmetry code: (i) $x+1, y, z$. 